\title{
Transactivating Target Gene Expression by Recombinant SALL4B: A Pluripotent Stem Cell Marker
}

\author{
Mengru Yuan ${ }^{1 \#}$, Yun Wang ${ }^{2 \#}$, Zhihua Ren ${ }^{1,3}$, Wei Dai ${ }^{1,4 *}$ and Yongping Jiang ${ }^{1,3 *}$ \\ ${ }^{1}$ Biopharmaceutical R\&D Center, Chinese Academy of Medical Sciences \& Peking Union Medical College, Suzhou, China \\ ${ }^{2}$ Obstetrical Department, Suzhou Municipal Hospital Affiliated Nanjing, Medical University, Suzhou, China \\ ${ }^{3}$ Biopharmagen. Corp. Suzhou, China \\ ${ }^{4}$ New York University, Langone Medical Center, New York, USA \\ \#These authors contributed equally to this study
}

\begin{abstract}
SALL4 is an important transcription factor that supports the expansion of hematopoietic stem cells. SALL4 expression is also deregulated in several types of leukemia. Recent studies reveal that SALL4B, a major isoform of SALL4, is heavily modified by post-translational mechanisms and these modifications are critical for their stability, subcellular localization, and transcriptional activities. Given the importance of SALL4B in supporting stem cell selfrenewal and expansion, we optimized a large scale expression and purification process to obtain SALL4B using the baculovirus expression vector system. Recombinant TAT-SALL4B was efficiently purified by nickel affinity chromatography under native conditions. Immuno-blotting confirmed that recombinant SALL4B was highly expressed and purified. As the first step to test the biological activity of purified TAT-SALL4B, we investigated whether TATSALL4B, directly supplemented to the culture medium, was capable of entering into cells through the protein transduction process. Fluorescent microscopy revealed that recombinant TAT-SALL4B specifically localized to the nucleus in a concentration- and time-dependent manner. Reporter gene assays showed that purified TAT-SALL4B protein activated OCT4 gene promoter, indicating that recombinant SALL4B was transcriptionally active in vivo. Combined, our results suggest that TAT-SALL4B may provide a promising factor for supporting ex vivo expansion of hematopoietic stem cells.
\end{abstract}

Keywords: Baculoviral expression system; Protein purification; Reporter gene; TAT-SALL4B; Transcriptional activation

\section{Introduction}

Over the past decade, hematopoietic stem cell transplantation has become the standard treatment for many human malignancies including multiple myeloma and acute myeloid leukemia [1-3]. The success of stem cell transplantation therapy greatly relies on efficient expansion of hematopoietic stem cells, including umbilical cord blood stem cells, without loss of pluripotency [4-6]. SALL4 is a zinc-finger transcriptional factor that plays a critical role in the maintenance of stem cells pluripotency by physical and functional interaction with stem cell factors such as OCT4, NANOG, and SOX2 [7-10]. SALL4 exists as two isoforms (SALL4A and SALL4B) and both isoforms are detected in many pluripotency loci in embryonic stem cells [11]. Recent studies show that infection with either SALL4A- or SALL4Blentivirus dramatically expand human hematopoietic stem cells ex vivo and enhance their ability of long-term engraftment in NOD/ SCID mice [12-14]. However, the potential risk of non-regulatable transgene expression and the emergence of replication-competent retrovirus limit its clinical application $[15,16]$. Recently, attempts have been made to deliver bioactive heterogeneous proteins into the cell via cell-penetrating peptides because of the safety consideration [17]. TAT-linked peptides facilitate their transduction into cells via lipid raft-dependent macropinocytosis, which has generated considerable interests for protein therapeutics [18-21].

Among SALL4 protein family, SALL4B is a more prevalent form in transformed cell lines, as well as in mouse stem cells. For example, SALL4A expression is barely detectable in Tera-1 and NT2/D1 cells whereas SALL4B is highly expressed in these cells [22]. Furthermore, a recent study has demonstrated that SALL4B can protect mice exposed to lethal irradiation and enhance both short-term and long-term engraftment of hematopoietic stem cell expansion in NOD/SCID mice [23].
Given the importance of SALL4B, we produced a large quantity of TAT-SALL4B protein through the baculovirus expression vector system. This expression system offers the advantage of posttranslational modifications of heterologous proteins in a manner similar to those observed in mammalian cells [24]. A recent study shows that post-translational modifications are known to be essential for the stability, subcellular localization, and transcriptional activities of SALL4B [25]. In the current study, we established a large-scale expression and purification system for recombinant TAT-SALL4B. We confirmed that the purified recombinant TAT-SALL4B was biologically active. Our study thus provides an excellent platform for the potential use of SALL4B to expand hematopoietic stem cells for future clinical applications.

\section{Methods}

\section{Expression of TAT-SALL4B in Sf9 insect cells}

SALL4B cDNA was cloned into pFastBac ${ }^{\mathrm{TM}}$ vector (Invitrogen) which contains a polyhedrin promoter for high expression of recombinant protein. A TAT sequence and six histidines were inserted in-frame in the N-terminus. Sf9 (Spodoptera frugiperda) cells were

*Corresponding author: Yongping Jiang, Biopharmaceutical R\&D Center, Chinese Academy of Medical Sciences \& Peking Union Medical College, Suzhou, China Tel: 86-512-62831269; Fax: 86-512-62831219; E-mail: yjiang@biopharmagen. com, wei.dai@nyumc.org

Received March 10, 2014; Accepted May 05, 2014; Published May 07, 2014

Citation: Yuan M, Wang Y, Ren Z, Dai W, Jiang Y (2014) Transactivating Target Gene Expression by Recombinant SALL4B: A Pluripotent Stem Cell Marker. J Stem Cell Res Ther 4: 203. doi:10.4172/2157-7633.1000203

Copyright: ( 2014 Yuan M, et al. This is an open-access article distributed unde the terms of the Creative Commons Attribution License, which permits unrestricted use, distribution, and reproduction in any medium, provided the original author and source are credited. 
cultured in Sf-900 $27^{\circ} \mathrm{C} \pm 0.5^{\circ} \mathrm{C}$ with gentle agitation. After reaching a density of $2 \times 10^{6}$ cells $/ \mathrm{ml}, S f 9$ cells were infected with recombinant baculovirus carrying human SALL4B gene. Cells were harvested on day 3 post-infection. The supernatant was filtered through $0.45 \mu \mathrm{m}$ filter and saved as a viral stock. Plaque assay was performed on the viral stock to determine titer. Cells were resuspended and lysed in 10\% SDS-PAGE sample buffer. Equal amounts of samples were blotted with both anti-His (Santa Cruz Biotech) and anti-SALL4 antibodies (Abcom) as primary antibody, followed by the secondary antibody Ig $G$ conjugated to alkaline phosphatase (Biolegend).

\section{Optimizing expression of TAT-SALL4B}

Sf9 cells were harvested at 3-7 days post-infection and cell pellets were lysed and analyzed by Western blot with an antibody to SALL4 at 1:1000 dilution. The same method was used to detect SALL4B expression efficiency affected by different $S f 9$ cell densities $\left(3.2 \times 10^{4}\right.$ cells $/ \mathrm{ml}-6.5 \times 10^{6}$ cells $/ \mathrm{ml}$, accordingly M.O.I=20-0.1) before infection.

\section{Purification of TAT-SALL4B protein}

Recombinant TAT-SALL4B protein was purified by nickel affinity chromatography under a native condition. Baculovirus-infected $S f g$ cells pellets were thawed and chilled on ice and then resuspended in the lysis buffer containing $50 \mathrm{mM} \mathrm{NaH}_{2} \mathrm{PO}_{4}, 500 \mathrm{mM} \mathrm{NaCl}, 1 \% \mathrm{CA} 630$ and protease inhibitor ( $\mathrm{pH}$ 8.0). The suspension was sonicated on ice (20 rounds) and centrifuged at 10,000 rpm for $15 \mathrm{~min}$. The supernatant was collected, which was then supplemented with Ni-NTA (Qiagen) resin slurry. The mixture was gently agitated on a rotary shaker for $1 \mathrm{~h}$. Resin was collected by centrifugation and rinsed with a washing buffer containing $25 \mathrm{mM}$ imidazole, $50 \mathrm{mM} \mathrm{NaH}_{2} \mathrm{PO}_{4}$, and $500 \mathrm{mM}$ $\mathrm{NaCl}$ ( $\mathrm{pH}$ 8.0) until little proteins were present in the supernatant as determined by the Bradford assay. Proteins specifically bound to the resin were eluted by the washing buffer containing $250 \mathrm{mM}$ imidazole. All the purification processes were carried out at $4^{\circ} \mathrm{C}$. Fractions in all steps were collected and analyzed by SDS-PAGE and Western blotting to monitor the recovery of TAT-SALL4B. Eluted TAT-SALL4B was dialysis in a buffer containing 5\% glycerol, $25 \mathrm{mM}$ Tris, $5 \mathrm{mM}$ EDTA, and $2 \mathrm{mM}$ DTT for $16 \mathrm{~h}$. The final purified recombinant protein was stored in $-80^{\circ} \mathrm{C}$ for various analyses.

\section{TAT-SALL4B protein transduction}

HeLa cells were seeded in 24-well plates at a density of about 1,000 cells/well. After overnight culture, the culture medium was removed and supplemented with serum-free medium for $6 \mathrm{~h}$. These cells were then cultured in the normal medium supplemented with $100 \mathrm{nM}$ or $500 \mathrm{nM}$ TAT-SALL4B protein and incubated in $37^{\circ} \mathrm{C}$ for either $1.5 \mathrm{~h}$ or $8 \mathrm{~h}$. Control groups were supplemented with $500 \mathrm{nM}$ BSA or the mock eluted fraction of uninfected Sf9 cells. At the end of culture, cells were fixed with $4 \%$ paraformaldehyde, permeabilized with $0.25 \%$ TritonX-100, and blocked with $5 \%$ serum for $1 \mathrm{~h}$. The cells were then incubated with the anti-SALL4 antibody (1:800) overnight at $4^{\circ} \mathrm{C}$ and then with a Cy3-conjugated secondary antibody (Jackson ImmunoResearch, 1:500) for $1 \mathrm{~h}$ in $37^{\circ} \mathrm{C}$. Cells at each step were washed with PBS. DNA was finally stained with Hoechst (Beyotime) for 5 min. HeLa cells were examined by fluorescence microscopy.

\section{Dual luciferase reporter assay of TAT-SALL4B protein}

HEK293T cells were seeded in 24-well plates at a density of 10,000 cells/well. After overnight culture, these cells were transfected with plasmid constructs expressing OCT4-driven luciferase using
Lipofectamin 2000 (Invitrogen) according to the manufacturer's instruction. Renilla-luciferase expression plasmid was used for co-transfection for normalizing transfection efficiency. After 12 $\mathrm{h}$ transfection, HEK293T cells were replaced with fresh medium containing different concentrations $(5 \mathrm{nM}, 10 \mathrm{nM}, 50 \mathrm{nM}, 100$ $\mathrm{nM}$ ) of purified recombinant TAT-SALL4B. Control groups were supplemented with either $500 \mathrm{nM}$ BSA or various concentrations of heat-inactivated SALL4B protein $(50 \mathrm{nM}$ and $100 \mathrm{nM})$. These cells were cultured for $24 \mathrm{~h}$ and then supplemented with an additional batch of recombinant TAT-SALL4B or control proteins. After other $24 \mathrm{~h}$ the luciferase activity was analyzed using a dual luciferase reporter assay kit according the instruction provided by the supplier (Promega).

\section{Results}

\section{Expression of TAT-SALL4B in Sf9 insect cells}

Given the importance of SALL4 in the maintenance of pluripotency of stem cells through regulating OCT4 expression [8], we attempted to obtain a large of recombinant human TAT-SALL4B that could be used for protein transduction. We infected $S f 9$ cells with recombinant baculovirus expressing His-TAT-SALL4B (Figure 1A). We noticed that morphology of $S f 9$ cells underwent dramatic changes after infection with recombinant baculoviruses. Specifically, the size of $S f g$ cells greatly increased $72 \mathrm{~h}$ post-infection with the presence of vesicular bodies in the cytoplasm (Figure 1B). These cells started to lyse at about $120 \mathrm{~h}$ post-infection. Sfg cells were collected and analyzed by SDS-PAGE. The Coomassie blue staining was not sufficient for indicating the presence of SALL4B. However, after affinity purification we were able to detect proteins that are significantly enriched by $\mathrm{Ni}$ resin (Figure 1C). The enriched protein had a molecular weight of $95 \mathrm{kDa}$, which is the same as reported for ubiquitinated form of SALL4B [25]. We also noticed a band with a molecular weight of $70 \mathrm{kDa}$, which is the same

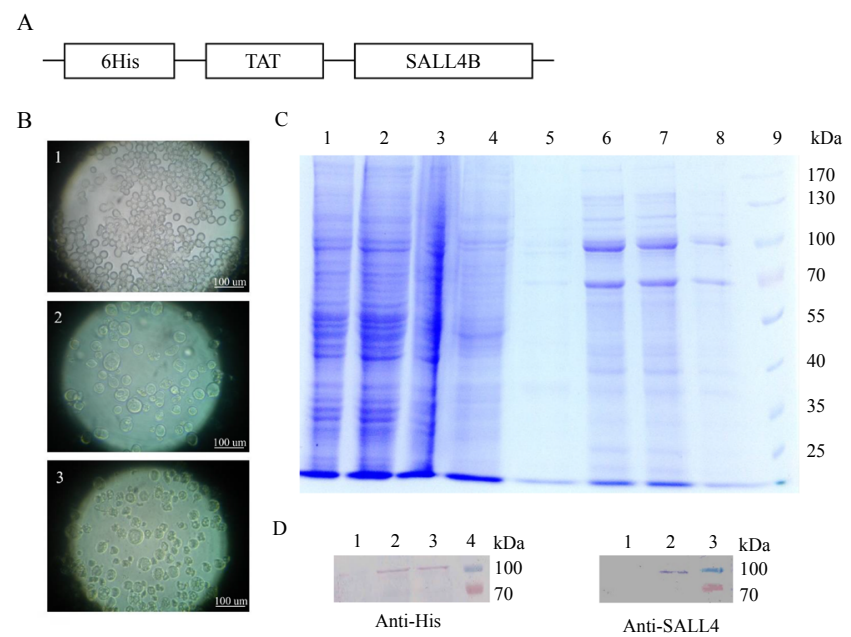

Figure 1: Expression and purification of TAT-SALL4B protein. (A) Schematic illustration of TAT-SALL4B fusion protein construct. (B) Sf9 cell morphology at different times post-infection. 1: uninfected Sf9 cell. 2: Sf9 cells at $72 \mathrm{~h}$ post-infection. 3: Sf9 cells at $120 \mathrm{~h}$ post-infection. (C) Purified TATSALL4B was resolved on a $10 \%$ denaturing gel and stained with Coommasie brilliant blue. Lane 1: lysates of uninfected Sf9 cells. Lane 2: lysates of infected Sf9 cells. Lane 3: flow through fraction. Lane 4 and 5: wash fractions. Lanes 6, 7, 8: elution fractions 1, 2, 3. Lane 9: molecular markers. (D) Western blot analysis with anti-His antibody. Lane 1: uninfected Sf9 cells. Lane 2, 3: Infected Sf9 cells. Lane 4: molecular markers. (E) Western blot analysis with anti-SALL4 antibody. Lane 1: uninfected Sf9 cells. Lane 2: Infected Sf9 cells. Lane 3: molecular markers. 
as unmodified SALL4B [25]. These two bands were absent in the lanes of uninfected cells. Expression of SALL4B was further confirmed by Western blotting with antibodies to both His tag and SALL4 (Figure $1 \mathrm{D}$ and $1 \mathrm{E})$.

\section{Optimizing expression and purification of TAT-SALL4B}

We noticed that the expression level of SALL4B varied greatly due to the cell density, as well as the length of infection. The expression level was highest at day 3 to 5 post infection (Figure 2A). Likewise, SALL4B expression was high with a seeding cell density of about $3.2 \times 10^{5}-6.5 \times 10^{5}$ cells $/ \mathrm{ml}$ (Figure $2 \mathrm{~B}$ ). Because of a significant loss of recombinant SALL4B during purification, we also optimized the purification process. We investigated many different lysis methods and found that most of these methods caused significant loss of the recombinant protein. We finally settled at the purification method as described in the Methods that appeared to completely release SALL4B protein to cell lysates (Figure 2C).

\section{TAT-SALL4B protein transduction}

Protein transduction using membrane-penetrating peptides has been reported [18-21]. This method holds a great promise to both clinical application and basic research because it is capable of efficiently deliver heterogeneous proteins into the cells. It is known that the nuclear localization relies on the presence of a nuclear localization signal [26-28]. As a transcription factor, SALL4B needs to localize to the nucleus in order to regulate expression of target genes. As the first step to test the biological activity of purified TAT-SALL4B, we investigated whether TAT-SALL4B that was directly supplemented to the culture medium was capable of entering into HeLa cells. Fluorescence microscopy showed that specific signals were present in some of the cells transduced with SALL4B protein. The signal intensity and the number of cells containing the signals were both concentration- and time-dependent (Figure 3). Cells transduced with mock proteins did not exhibit any signals after staining with the SALL4 antibody which is a commercially available antibody for fluorescence staining.

A
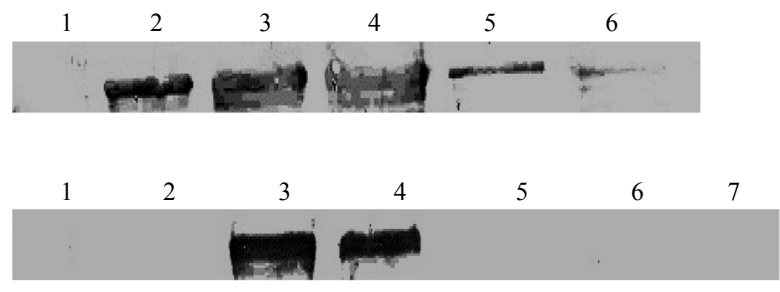

$\mathrm{C}$

\begin{tabular}{ccccccc}
1 & 2 & 3 & 4 & 5 & 6 & 7 \\
\hdashline
\end{tabular}

Figure 2: Optimizing expression and purification of TAT-SALL4B. Western blot analysis of expression efficiency of TAT-SALL4B protein affected by days of infection and initial cell density. (A) Lane1: pellets from uninfected Sf9 cells. Lane2-6: Sf9 cells pellet at 3-7 days post-infection. (B) Lane1-6: Sf9 cell pellets at day 3 post-infection with different initial cell densities $(3.2 \times 104$ cells $/ \mathrm{ml}, 6.5 \times 104$ cells $/ \mathrm{ml}, 3.2 \times 105$ cells $/ \mathrm{ml}, 6.5 \times 105$ cells $/ \mathrm{ml}, 3.2 \times 106$ cells/ $\mathrm{ml}$, and $6.5 \times 106$ cells $/ \mathrm{ml}$; M.O.I was $20,10,2,1,0.2$, and 0.1 , respectively). Lane7: pellets from uninfected Sf9 cells. (C) Lane1: precipitates of lysed uninfected Sf9 cells. Lane2: precipitates of lysed infected Sf9 cells. Lane3: lysates of uninfected Sf9 cells. Lane4: lysates of infected Sf9 cells. Lane 5: flow through fraction. Lane 6, 7: eluted fractions.

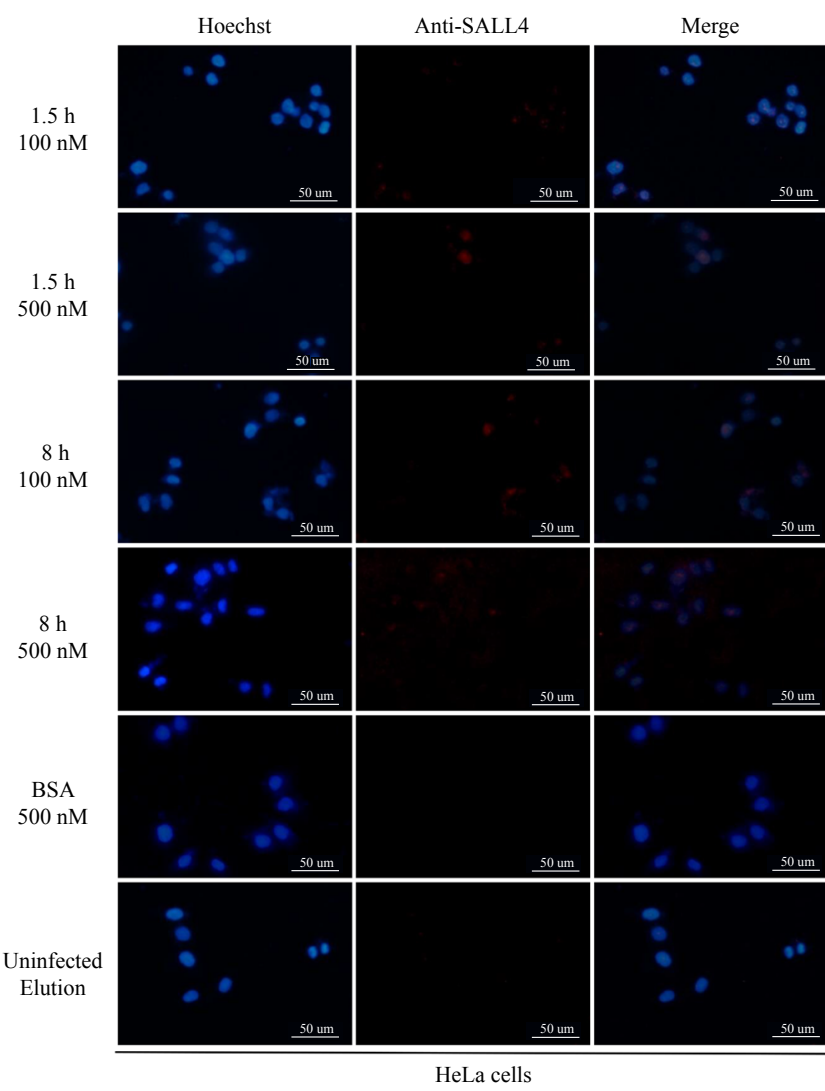

Figure 3: Characterization of TAT-mediated transduction of SALL4B protein. HeLa cells were cultured in the medium containing $100 \mathrm{nM}$ or 500 $\mathrm{nM}$ of TAT-SALL4B protein and incubated in $370 \mathrm{C}$ for either $1.5 \mathrm{~h}$ or $8 \mathrm{~h}$. Two control groups were included and they were $500 \mathrm{nM} \mathrm{BSA}$ and the mock eluted fraction of uninfected Sf9 cells. After incubation, protein transduction was assayed by immunofluorescence staining with the anti-SALL4 antibody and Cy3-conjugated secondary antibody (red). Hoechst was used to stain the cell nuclei (blue). The merges images revealed that recombinant TAT-SALL4B could transduce into cells and specifically localized to the nucleus. Original magnification $\times 200$.

\section{Regulating target gene expression by TAT-SALL4B protein}

SALL4 plays a critical role in maintaining pluripotency of embryonic stem cells by regulating transcription of Pou $5 f 1$ which encodes OCT4 [8]. Unlike cobalt and nickel metals, OCT4 induction could be transcriptional activated by SALL4 [22]. Therefore, the activity of TAT-SALL4B protein could be studied in vivo by its ability to transactivate genes driven by the OCT4 promoter. To this end, we first co-transfected HEK293T cells with plasmids expressing OCT4-firefly luciferase reporter and renilla-luciferase and then added TAT-SALL4B of various concentrations to the medium of transfected cells (Figure $4 \mathrm{~A}$ for the treatment scheme). At the end of treatments, cells were collected and lysed. Equal amounts of cell lysates were analyzed for luciferase activities. We observed that purified TAT-SALL4B protein was capable of transactivating expression of firefly luciferase driven by the OCT4 promoter in a concentration-dependent manner (Figure 4B). From 10 nM SALL4B treatment, we observed significant increases in the reporter gene expression (compared to BSA, two-tailed Student's t-test, $P=0.001,0.04,0.001$, respectively). The activation of the reporter gene by TAT-SALL4B was specific as heat-inactivated TAT-SALL4B was incapable of transactivating luciferase expression. 
A

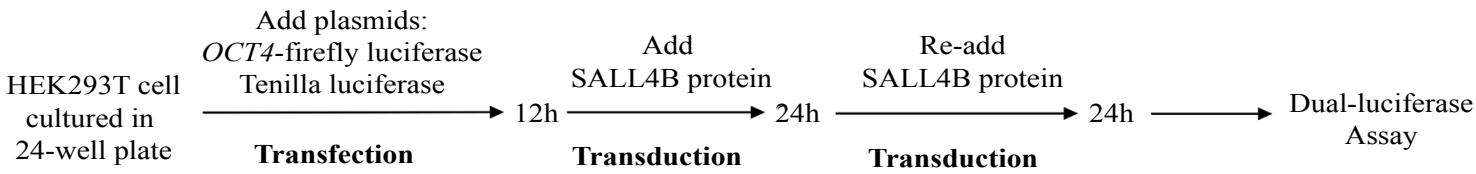

B

\section{OCT4 Promoter Luciferase}

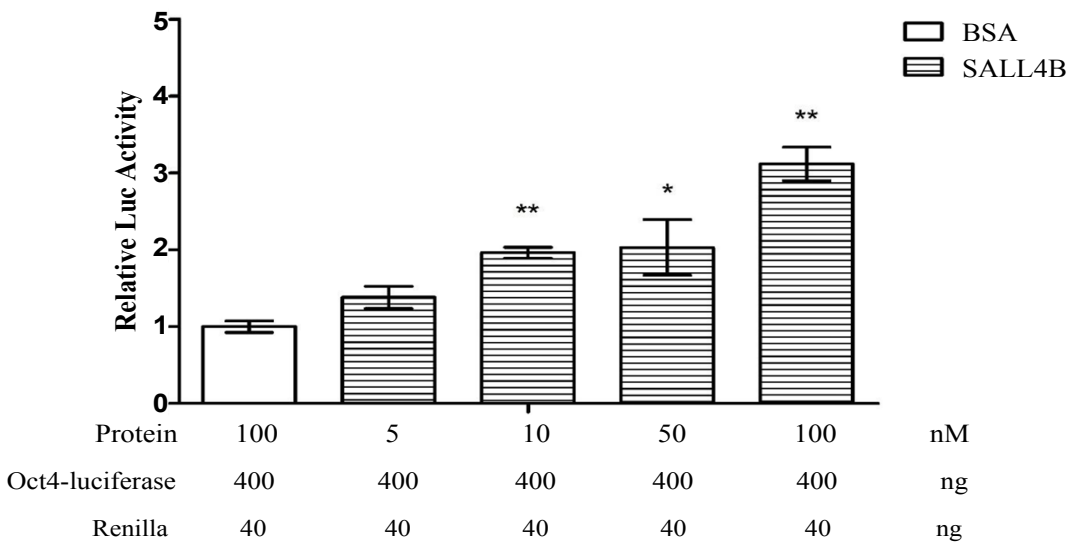

OCT4 Promoter Luciferase

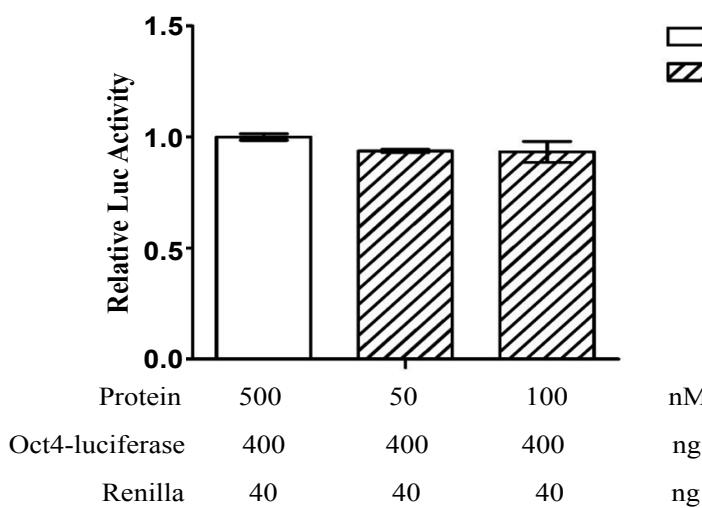

Figure 4: TAT-SALL4B was transcriptionally active in vivo. (A) Schematic diagram of experimental procedures. (B) After HEK293T cells were transfected with OCT4-driven luciferase and Renilla-luciferase expression plasmids, cells were cultured in the medium containing different concentrations (5, 10, $50,100,500 \mathrm{nM}$ ) of purified recombinant TAT-SALL4B for $48 \mathrm{~h}$. Control groups were supplemented with either BSA or various concentrations of heat-inactivated SALL4B (50, 100 nM). After incubation, cell lysates were prepared and assayed for firefly and renilla luciferase activities. Firefly luciferase activity was normalized to renilla luciferase activity and the activity of the control group supplemented with BSA was set as 1.0. Bars represent the mean of 3 replicates with standard derivation. The symbol ** means $P$ $<0.01$ and * means $\mathrm{P}<0.05$ as determined by two-tailed Student's t-test. The data were representative of two independent experiments.

\section{Discussion}

SALL4B is an important stem cell transcription factor, which has an essential impact on the self-renewal of hematopoietic stem cell [29]. Several other investigations have been focused on expressing SALL4B protein. In the current study, we primarily used a eukaryotic expression system (baculoviral expression system) whereas Liao et al. [12] used the prokaryotic (E. coli) expression system. SALL4B expressed by E. coli is largely insoluble (inclusion body). In our system, SALL4B is soluble and in a native confirmation when purified through nickel resin. Compared to Liao et al. [23] we elaborated the expression and purification process in detail. Furthermore, we have optimized the system to obscure maximum yield of the recombinant TAT-SALL4B. The fluorescence staining results showed that recombinant TAT-SALL4B protein we produced could enter the cells and specifically localize to the nucleus. As SALL4B's concentration or the time of treatment increases, the fluorescence signal intensity and the total number of cells containing the signals both increased. The dual luciferase reporter assay revealed that the purified TAT-SALL4B protein is capable of activating target gene expression in vivo. We further analyzed expression of Nanog in the transfected cells and did not see a significant change of its expression. We did not observe the modulation of endogenous SALL4 either. This might be because the percentage of cells transduced with 
Citation: Yuan M, Wang Y, Ren Z, Dai W, Jiang Y (2014) Transactivating Target Gene Expression by Recombinant SALL4B: A Pluripotent Stem Cell Marker. J Stem Cell Res Ther 4: 203. doi:10.4172/2157-7633.1000203

Page 5 of 5

SALL4B recombinant protein remained low. However, our study provides proof-of-concept evidence that nuclear transcription factors can be delivered to the cell, altering gene expression. This is of great significance because of the following reasons. (1) This approach does not perturb the genome, thus causing no genomic instability. (2) SALL4 is an important regulator of OCT4, the latter is known as the master regulator of stem cell self-renewal and expansion. It is conceivable that OCT4 gene expression can be manipulated through the use of TAT-SALL4B. (3) More importantly, TAT-SALL4B can be used for ex vivo hematopoietic stem cell expansion. These expanded cells will be valuable for the treatment of various hematopoietic diseases in the clinics.

\section{Acknowledgment}

This work was supported by key State projects of China (2011ZX09102-01004 and 2011ZX09401-027)

\section{Disclosure}

All authors have no conflict of interest to disclose.

\section{References}

1. Attal M, Harousseau JL, Stoppa AM, Sotto JJ, Fuzibet JG et al. (1996) A prospective, randomized trial of autologous bone marrow transplantation and chemotherapy in multiple myeloma. N Engl J Med 335(2): 91-97.[PubMed]

2. Child JA, Morgan GJ, Davies FE, Owen RG, Bell SE, et al. (2003) High-dose chemotherapy with hematopoietic stem-cell rescue for multiple myeloma. $\mathrm{N}$ Engl J Med 348(19): 1875-1883.[PubMed]

3. Woods WG, Neudorf S, Gold S, Sanders J, Buckley JD, et al. (2001) A comparison of allogeneic bone marrow transplantation, autologous bone marrow transplantation, and aggressive chemotherapy in children with acute myeloid leukemia in remission: a report from the Childrenss Cancer Group. Blood 97(1): 56-62.[PubMed]

4. Wagner JE, Barker JN, DeFor TE, Baker KS, Blazar BR, et al. (2002) Transplantation of unrelated donor umbilical cord blood in 102 patients with malignant and nonmalignant diseases: influence of CD34 cell dose and HLA disparity on treatment-related mortality and survival. Blood 100(5): 1611-1618. [PubMed]

5. Gluckman E, Rocha V, Boyer-Chammard A, , Locatelli F, Arcese W, et al. (1997) Outcome of cord-blood transplantation from related and unrelated donors. Eurocord Transplant Group and the European Blood and Marrow Transplantation Group. N Engl J Med 337(6): 373-381.[PubMed]

6. Laughlin MJ, Barker J, Bambach B, Koc ON, Rizzieri DA, et al. (2001) Hematopoietic engraftment and survival in adult recipients of umbilical-cord blood from unrelated donors. N Engl J Med 344(24): 1815-1822.[PubMed]

7. Yang J, Chai L, Fowles TC, Alipio Z, Xu D, et al.(2008) Genome-wide analysis reveals Sall4 to be a major regulator of pluripotency in murine-embryonic stem cells. Proc Natl Acad Sci U S A 105(50): 19756-1961.[PubMed]

8. Zhang J, Tam WL, Tong GQ, Wu Q, Chan HY, et al. (2006) Sall4 modulates embryonic stem cell pluripotency and early embryonic development by the transcriptional regulation of Pou5f1. Nat Cell Biol 8(10): 1114-1123.[PubMed]

9. Wu Q, Chen X, Zhang J, Loh YH, Low TY, et al. (2006) Sall4 interacts with Nanog and co-occupies Nanog genomic sites in embryonic stem cells. J Biol Chem 281(34): 24090-24094.[PubMed]

10. Orkin SH, Hochedlinger K (2011) Chromatin connections to pluripotency and cellular reprogramming. Cell 145(6): 835-850.[PubMed]
11. Rao S, Zhen S, Roumiantsev S, McDonald LT, Yuan G-C et al (2010) Differential roles of Sall4 isoforms in embryonic stem cell pluripotency Molecular and cellular biology 30(22):5364-5380.[PubMed]

12. Aguila JR, Liao W, Yang J, Avila C, Hagag N, et al. (2011) SALL4 is a robust stimulator for the expansion of hematopoietic stem cells. Blood 118(3): 576585.[PubMed]

13. Yang J, Aguila JR, Alipio Z, Lai R, Fink LM, et al. (2011) Enhanced self-renewa of hematopoietic stem/progenitor cells mediated by the stem cell gene Sall4. J Hematol Oncol 4: 38.[PubMed]

14. Aguila JR, Mynarcik DC, Ma Y (2011) SALL4: finally an answer to the problem of expansion of hematopoietic stem cells? Expert Rev Hematol 4(5): 479-481. [PubMed]

15. Pluta K, Luce MJ, Bao L, Agha-Mohammadi S, Reiser J (2005) Tight control of transgene expression by lentivirus vectors containing second-generation tetracycline-responsive promoters. J Gene Med 7(6): 803-817.[PubMed]

16. Wu X, Wakefield JK, Liu H, Xiao H, Kralovics R, et al. (2000) Development of a novel trans-lentiviral vector that affords predictable safety. Mol Ther 2(1): 47-55.[PubMed]

17. Gait MJ (2003) Peptide-mediated cellular delivery of antisense oligonucleotides and their analogues. Cell Mol Life Sci 60(5): 844-853.[PubMed]

18. Wadia JS, Stan RV, Dowdy SF (2004) Transducible TAT-HA fusogenic peptide enhances escape of TAT-fusion proteins after lipid raft macropinocytosis. Nat Med 10(3): 310-315.[PubMed]

19. Schwarze SR, Ho A, Vocero-Akbani A, Dowdy SF (1999) In vivo protein transduction: delivery of a biologically active protein into the mouse. Science 285(5433): 1569-1572.[PubMed]

20. Nagahara H, Vocero-Akbani AM, Snyder EL, , Ho A, Latham DG, et al. (1998) Transduction of full-length TAT fusion proteins into mammalian cells: TATp27Kip1 induces cell migration. Nat Med 4(12): 1449-1452.[PubMed]

21. Zhao M, Weissleder R (2004) Intracellular cargo delivery using tat peptide and derivatives. Med Res Rev 24(1): 1-12.[PubMed]

22. Yao Y, Lu Y, Chen WC, Jiang Y, Cheng T, Ma Y, et al. (2014) Cobalt and nicke stabilize stem cell transcription factor OCT4 through modulating its sumoylation and ubiquitination. PLoS One 9(1): e86620.[PubMed]

23. Liao W, Aguila JR, Yao Y, Yang J, Zieve G, et al. (2013) Enhancing bone marrow regeneration by SALL4 protein. J Hematol Oncol 6(1): 84.[PubMed]

24. Jarvis DL (1997) Baculovirus expression vectors. In: L.K Miller (ed.)The Baculoviruses. Plenum Press, New York, 389-431.

25. Yang F, Yao Y, Jiang Y, Lu L, Ma Y, et al. (2012) Sumoylation is important for stability, subcellular localization, and transcriptional activity of SALL4, an essential stem cell transcription factor. J Biol Chem 287(46): 38600-38608. [PubMed]

26. Li J, Pan G, Cui K, Liu Y, Xu S, et al. (2007) A dominant-negative form of mouse SOX2 induces trophectoderm differentiation and progressive polyploidy in mouse embryonic stem cells. J Biol Chem 282(27): 19481-19492.[PubMed]

27. Pan G, Qin B, Liu N, Schöler HR, Pei D (2004) Identification of a nuclear localization signal in OCT4 and generation of a dominant negative mutant by its ablation. J Biol Chem 279(35): 37013-37020.[PubMed]

28. Bartholomeusz G, Talpaz M, Bornmann W, Kong LY, Donato NJ (2007) Degrasyn activates proteasomal-dependent degradation of c-Myc. Cancer Res 67(8): 3912-3918.[PubMed]

29. Ma Y, Cui W, Yang J, Qu J, Di C, et al. (2006) SALL4, a novel oncogene, is constitutively expressed in human acute myeloid leukemia (AML) and induces AML in transgenic mice. Blood 108(8): 2726-2735.[PubMed]. 